\title{
The Use of Compression Measurements to Study Stress-Strain Relationships and the Volume Dependence of the Elastic Free Energy of a Polymer Network
}

\author{
Chin U. YU and James E. MARK ${ }^{* \dagger}$ \\ Department of Chemistry and the Macromolecular Research Center, \\ The University of Michigan, Ann Arbor, Michigan, 48104, USA.
}

(Received July 31, 1974)

\begin{abstract}
Poly(dimethylsiloxane) networks were prepared by cross-linking the polymer both in the undiluted state and in cyclohexane solutions of varying composition. Stress-strain isotherms of these networks in compression in the unswollen state were found to show only small deviations from the form predicted by the statistical theories of rubberlike elasticity. The dependence of these deviations on the variables which characterize the preparation of these networks indicates that they are not due primarily to nonequilibrium effects. The dependence of the stress itself on the volume at which the networks were prepared is in close agreement with the Flory theory of rubberlike elasticity, which contains a volume-dependent contribution to the elastic free energy of a polymer network. Previous experimental results bearing on the existence and magnitude of such a contribution are critically summarized and reevaluated, thereby definitively settling an important controversy in the area of rubberlike elasticity.

KEY WORDS Cross-Linking / Stress-Strain Isotherms / Compression / Mooney-Rivlin Constants / Elastic Free Energy / Polymer Networks / Rubberlike Elasticity /
\end{abstract}

In general, studies of the stress-strain behavior of rubberlike networks have used uniaxial elongation as the deformation; comparatively little work has been carried out on networks in compression. ${ }^{1-3}$ In this present study, we report stress-strain relationships for poly(dimethylsiloxane) (PDMS) networks prepared both in the undiluted state and in solution. It has previously been shown $\mathrm{n}^{4,5}$ that such variation in the conditions of cross-linking very significantly affects both equilibrium and nonequilibrium properties of polymer networks in elongation, and the extension of such studies to networks in compression is obviously of considerable interest. Most importantly, compression measurements may be used to obtain the dependence of the stress exhibited by a network on the

$\uparrow$ To whom correspondence should be addressed. Permanent address: See above.

* Present address (Sept. 1, 1974-Jan. 8, 1975): Dept. of Chemical Engineering, Massachusetts Institute of Technology, Cambridge, Mass., 02139, USA. volume at which the network was formed. The nature of this dependence for networks in compression is of great interest, since current theories of rubberlike elasticity differ significantly in this respect. ${ }^{6,7}$

The quantity of interest in regard to these cited goals is the "reduced force" $\left[f^{*}\right]$, defined by

$$
\left[f^{*}\right]=f^{*} /\left(\alpha-\alpha^{-2}\right)
$$

where $f^{*}$ is the force or stress per unit area of the unswollen, undeformed sample and $\alpha$ (variously called the relative length, deformation, or compression) is the ratio of the length of the stressed sample to the length of the unstressed, isotropic sample at the same volume. ${ }^{8}$ According to the molecular or statistical theories of rubberlike elasticity, ${ }^{6,7}$ the reduced force is given by the expression ${ }^{7-10}$

$$
\left[f^{*}\right]=\nu k T v_{2, \mathrm{C}}^{2 / 3}
$$

where $\nu$ is the number of chains per unit volume of the network, $k$ is the Boltzmann constant, $T$ is the absolute temperature, and $v_{2, \mathrm{C}}$ is the 
volume fraction of polymer chains in the system being cross-linked which are successfully incorporated into the network structure. Theory thus predicts that, at constant temperature, the reduced force exhibited by a particular polymer network should be independent of $\alpha$. Actual stress-strain isotherms for uniaxial deformation are almost invariably, however, better represented by the empirical Mooney-Rivlin equation ${ }^{11}$

$$
\left[f^{*}\right]=2 C_{1}+2 C_{2} \alpha^{-1}
$$

The quantities $2 C_{1}$ and $2 C_{2}$ are constants, with the latter serving as a measure of the departure of the observed stress-strain relationship from that predicted by the molecular theories. For this reason, values of $2 C_{2}$ have been widely tabulated and discussed, ${ }^{1,12,13}$ and the dependence of this constant on the conditions of preparation of the network and the conditions used in its determination is a subject of continuing investigation. One aspect of particular interest in the present study is the fact that $2 C_{2}$ has generally been found to be very small in the case of networks in compression. ${ }^{2,3}$

Values of the reduced force are also used in the evaluation of the dependence of the stress on the volume at which the network was formed. Current theories differ in regard to this dependence, since there is considerable controversy over whether or not the elastic free energy of a polymer network should contain a logarithmic term in the volume $V .^{7-10,14-16}$ According to the theory of Flory ${ }^{8}$ such a term is required; its contribution to the free energy of the network is calculated to be $(\nu k T / 2) \ln V$, which is the free energy associated with the distribution of $\nu / 2$ cross-links over the sample volume $V$ to account for all the possible networks which might have been formed with the specified degree of cross-linking. James and Guth, ${ }^{14}$ however, argue that there is no basis for a term of this type. These two conflicting predictions may be tested by studying the stressstrain properties of several series of networks which have been prepared at different values of the volume fraction $v_{2, \mathrm{~s}}$ of polymer in the system being cross-linked, and which have been crosslinked to different extents (as measured by the volume fraction $v_{2, \mathrm{~N}}$ of polymer present in the network at swelling equilibrium in a thermodynamically good solvent at some convenient constant temperature). If $\left[f^{*}\right]_{\mathrm{B}}$ and $\left[f^{*}\right]_{\mathrm{S}}$ designate the reduced forces of a pair of networks exhibiting the same value of $v_{2, \mathrm{~N}}$ but prepared in the bulk (undiluted) state and in solution, respectively, then experimental values of $\left[f^{*}\right]_{\mathrm{s}} /$ $\left[f^{*}\right]_{\mathrm{B}}$ may be used as a direct test of the above theoretical predictions. As shown in detail elsewhere, ${ }^{7,9,10}$ the theory of Flory ${ }^{8}$ predicts that in the case of two such networks, for which $\left(v_{2, \mathrm{C}}\right)_{\mathrm{S}}<\left(v_{2, \mathrm{C}}\right)_{\mathrm{B}}$, the ratio $\left[f^{*}\right]_{\mathrm{S}} /\left[f^{*}\right]_{\mathrm{B}}$ should be significantly greater than unity. According to the theory of James and Guth, ${ }^{14}$ however, this ratio should be unity irrespective of the value of $v_{2, \mathrm{c}}$ characterizing the samples being compared. ${ }^{7,9,10}$ Since, as already mentioned, $\left[f^{*}\right]$ may be expected to show a small but possibly significant dependence on $\alpha$, it will also be useful to consider values of $2 C_{1}$, the value of $\left[f^{*}\right]$ extrapolated to $\alpha^{-1}=0$. Thus, experimental values of both $\left[f^{*}\right]_{\mathrm{S}} /\left[f^{*}\right]_{\mathrm{B}}$ and $\left(2 C_{1}\right)_{\mathrm{S}} /\left(2 C_{1}\right)_{\mathrm{B}}$ for networks in compression will be compared with the results predicted by the theories of Flory ${ }^{8}$ and James and Guth. ${ }^{14}$ In addition, values of $\left[f^{*}\right]_{\mathrm{S}} /\left[f^{*}\right]_{\mathrm{B}}$ obtained earlier in studies ${ }^{7,9,10}$ of networks in elongation will be summarized, and the results from these studies will be reevaluated by a calculation of the values of the alternate ratio $\left(2 C_{1}\right)_{\mathrm{S}} /\left(2 C_{1}\right)_{\mathrm{B}}$.

\section{EXPERIMENTAL}

The poly(dimethylsiloxane) (PDMS) networks were prepared at $25^{\circ} \mathrm{C}$, using $\gamma$-radiation in exactly the same manner as described previously. ${ }^{3}$ The cylindrical molds used to contain the undiluted PDMS or the PDMS dissolved in cyclohexane were of such dimensions as to give samples, when unswollen, with diameters in the range $1.6-1.8 \mathrm{~cm}$ and lengths in the range $3.2-$ $4.2 \mathrm{~cm}$. Benzene was used to extract uncrosslinked material from each sample, and the weight of material removed was used to calculate the value of $v_{2, \mathrm{c}}$ for that particular network from the value of $v_{2, \mathrm{~s}}$ characterizing the system from which it was prepared. ${ }^{9,10,17}$ The equilibrium degree of swelling of each extracted network in cyclohexane at $25^{\circ} \mathrm{C}$ was obtained from the weights of the network before and after swelling to equilibrium, and was characterized by the volume fraction $v_{2, \mathrm{~N}}$ of polymer in the swollen 
network. ${ }^{7,9,10,17}$ (For the relatively thick samples used in compression measurements, such extraction procedures and swelling experiments frequently required $20-30$ days).

The stress-compression measurements were carried out on the PDMS networks in the unswollen state, at $25^{\circ} \mathrm{C}$, as described elsewhere. ${ }^{3}$ Stresses were found to be highly reproducible over the range of deformations $\alpha \cong 0.95-0.75$ employed in the determination of the desired stress-strain isotherms. In the case of compression measurements, however, a considerable time interval is frequently required to reach elastic equilibrium.

\section{RESULTS AND DISCUSSION}

Undiluted PDMS(B) and four solutions (S1S4) of PDMS in cyclohexane were used to prepare the series of networks described in the first

Table I. Characteristics of the PDMS Networks and Their Stress-Strain Properties in Compressiona

\begin{tabular}{|c|c|c|c|c|c|c|c|c|}
\hline Sample & $v_{2, \mathrm{~S}}$ & $\begin{array}{l}\text { Radiation } \\
\text { dose, Mrad }\end{array}$ & $v_{2, \mathrm{C}}$ & $v_{2, \mathrm{~N}}$ & $\begin{array}{c}{\left[f^{*}\right],{ }^{\mathrm{b}}} \\
\mathrm{N} \mathrm{mm}^{-2}\end{array}$ & $\begin{array}{c}2 C_{2}, \\
\mathrm{~N} \mathrm{~mm}^{-2}\end{array}$ & $\underset{\mathrm{hr}}{\mathrm{Av} t_{\mathrm{eq}}}$ & $\begin{array}{c}\mathrm{Av} \\
{\left[f^{*}\right]_{\mathrm{eq}}} \\
{\left[f^{*}\right]_{\mathrm{in}}}\end{array}$ \\
\hline B-1 & 1.00 & 1.37 & 0.902 & 0.0655 & 0.0383 & 0.0041 & 12.2 & 0.802 \\
\hline 2 & 1.00 & 1.52 & 0.924 & 0.0900 & 0.0702 & 0.0097 & 10.3 & 0.885 \\
\hline 3 & 1.00 & 1.59 & 0.931 & 0.0982 & 0.0777 & 0.0094 & 8.11 & 0.903 \\
\hline 4 & 1.00 & 1.62 & 0.934 & 0.0992 & 0.0824 & 0.0156 & 7.39 & 0.912 \\
\hline 5 & 1.00 & 1.70 & 0.947 & 0.108 & 0.0960 & 0.0213 & 6.75 & 0.932 \\
\hline 6 & 1.00 & 1.71 & 0.952 & 0.109 & 0.0969 & 0.0223 & 6.73 & 0.935 \\
\hline S1-1 & 0.58 & 1.86 & 0.535 & 0.0650 & 0.0479 & 0.0047 & 7.50 & 0.853 \\
\hline 2 & 0.58 & 2.42 & 0.550 & 0.0828 & 0.0670 & 0.0031 & 5.86 & 0.934 \\
\hline 3 & 0.58 & 3.02 & 0.552 & 0.0944 & 0.0823 & 0.0038 & 4.19 & 0.952 \\
\hline 4 & 0.58 & 3.37 & 0.553 & 0.0954 & 0.0841 & 0.0082 & 3.97 & 0.964 \\
\hline 5 & 0.58 & 4.04 & 0.556 & 0.105 & 0.102 & 0.0019 & 3.63 & 0.973 \\
\hline 6 & 0.58 & 4.52 & 0.559 & 0.114 & 0.125 & 0.0098 & 3.17 & 0.981 \\
\hline S2-1 & 0.45 & 2.65 & 0.421 & 0.0618 & 0.0410 & 0.0031 & 3.69 & 0.886 \\
\hline 2 & 0.45 & 3.73 & 0.424 & 0.0827 & 0.0746 & 0.0092 & 1.97 & 0.962 \\
\hline 3 & 0.45 & 5.18 & 0.428 & 0.0953 & 0.0915 & 0.0076 & 1.64 & 0.975 \\
\hline 4 & 0.45 & 6.18 & 0.429 & 0.0996 & 0.0957 & 0.0046 & 1.32 & 0.987 \\
\hline 5 & 0.45 & 7.71 & 0.431 & 0.107 & 0.110 & 0.0055 & 0.94 & 0.992 \\
\hline 6 & 0.45 & 7.98 & 0.433 & 0.110 & 0.116 & 0.0110 & 0.61 & 0.996 \\
\hline 7 & 0.45 & 8.30 & 0.435 & 0.113 & 0.120 & 0.0221 & 0.56 & 0.996 \\
\hline 8 & 0.45 & 9.76 & 0.436 & 0.118 & 0.125 & 0.0099 & 0.54 & 0.998 \\
\hline 9 & 0.45 & 10.8 & 0.438 & 0.120 & 0.132 & 0.0110 & 0.45 & 0.998 \\
\hline S3-1 & 0.40 & 4.37 & 0.361 & 0.0757 & 0.0609 & 0.0046 & 3.67 & 0.954 \\
\hline 2 & 0.40 & 6.08 & 0.371 & 0.0939 & 0.0804 & 0.0020 & 2.24 & 0.983 \\
\hline 3 & 0.40 & 10.5 & 0.378 & 0.100 & 0.0907 & 0.0032 & 1.93 & 0.993 \\
\hline 4 & 0.40 & 14.6 & 0.381 & 0.105 & 0.102 & 0.0013 & 1.58 & 0.995 \\
\hline 5 & 0.40 & 20.2 & 0.384 & 0.107 & 0.106 & 0.0009 & 1.14 & 0.998 \\
\hline 6 & 0.40 & 23.9 & 0.390 & 0.111 & 0.117 & 0.0028 & 0.94 & 0.999 \\
\hline S4-1 & 0.28 & 10.8 & 0.267 & 0.0853 & 0.0734 & 0.0030 & 2.06 & 0.983 \\
\hline 2 & 0.28 & 13.6 & 0.268 & 0.0860 & 0.0742 & 0.0067 & 1.80 & 0.988 \\
\hline 3 & 0.28 & 20.9 & 0.268 & 0.0865 & 0.0742 & 0.0029 & 1.19 & 0.994 \\
\hline 4 & 0.28 & 22.9 & 0.268 & 0.0900 & 0.0818 & 0.0061 & 1.11 & 0.997 \\
\hline 5 & 0.28 & 25.7 & 0.267 & 0.0914 & 0.0843 & 0.0050 & 1.03 & 0.998 \\
\hline 6 & 0.28 & 32.4 & 0.268 & 0.0954 & 0.0864 & 0.0048 & 0.94 & 0.998 \\
\hline 7 & 0.28 & 37.3 & 0.268 & 0.0984 & 0.0891 & 0.0057 & 0.40 & 0.999 \\
\hline 8 & 0.28 & 43.1 & 0.269 & 0.108 & 0.114 & 0.0035 & 0.30 & 1.000 \\
\hline
\end{tabular}

a Studied in the unswollen state, at $25^{\circ} \mathrm{C}$.

$\mathrm{b}$ The reduced force at a compression of $\alpha=0.800$. 


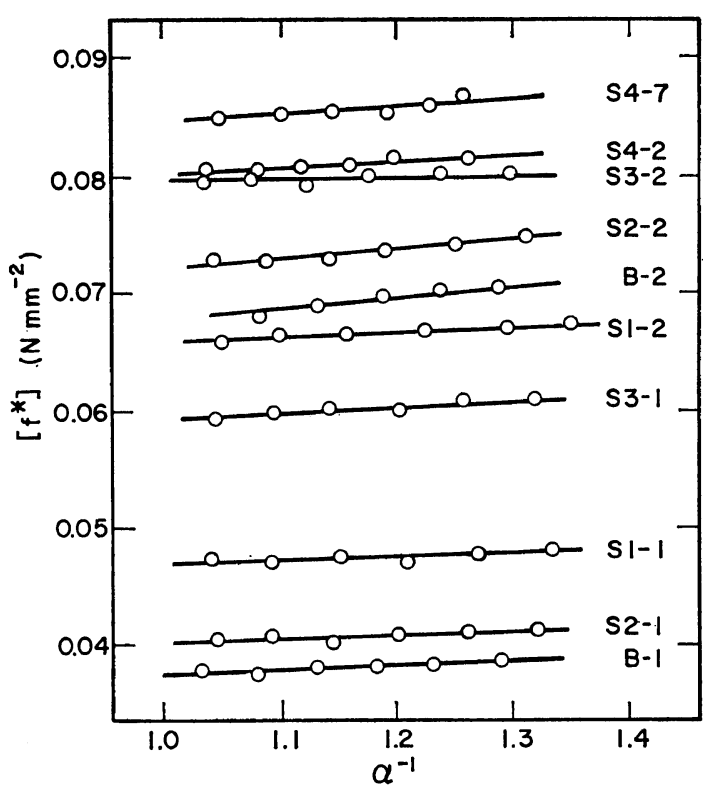

Figure 1. The dependence of the reduced force on the reciprocal compression for some typical unswollen poly(dimethylsiloxane) networks at $25^{\circ} \mathrm{C}$.

five columns of Table I. For convenience in interpretation, each network in a series prepared at a fixed value of $v_{2, \mathrm{~s}}$ was assigned the average value of $v_{2, \mathrm{C}}$ characterizing that series; for the series B, S1-S4, these values are $0.932( \pm 0.013)$, $0.551( \pm 0.005), 0.431( \pm 0.011), 0.378( \pm 0.010)$, and $0.268( \pm 0.001)$, respectively.

For each network, the reduced force was plotted against the reciprocal compression, as suggested by eq 3 ; typical results are presented in Figure 1. The relationship between $\left[f^{*}\right]$ and $\alpha^{-1}$ was found to be linear within experimental error, and the straight line representing each isotherm was located by the method of leastsquares. Each such isotherm is characterized in Table I by the reduced force at a compression of $\alpha=0.800$ and the slope of the line, designated by $2 C_{2}$ in eq 3 . (To aid in the comparison of these results with results from other studies, it should be noted that $1 \mathrm{~N} \mathrm{~mm}^{-2}=1 \mathrm{MNm}^{-2}=$ should be $10.20 \mathrm{~kg} \mathrm{~cm}^{-2}$ ). Typical values of $2 C_{2}$, those corresponding to $v_{2, \mathrm{~N}}=0.09$, are shown as a function of $v_{2, \mathrm{c}}$ in Figure 2; also included, for purposes of comparison, are corresponding results ${ }^{9}$ obtained on unswollen PDMS networks in elongation. These values of $2 C_{2}$ in compres-

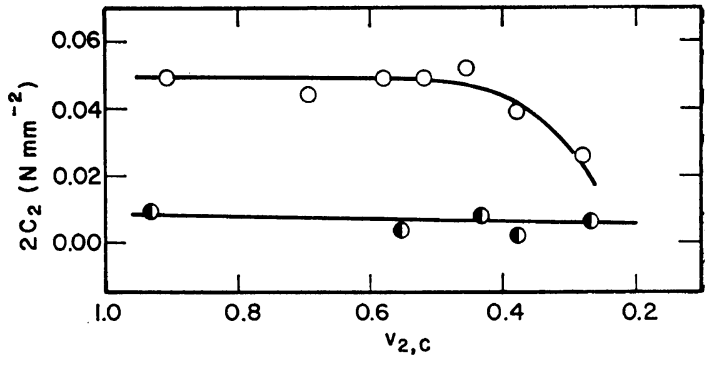

Figure 2. The dependence of the empirical constant $2 C_{2}$ on $v_{2, \mathrm{C}}$ (the volume fraction of PDMS chains in the system being cross-linked which are successfully incorporated into the network structure). Open circles refer to previously published results ${ }^{9}$ on PDMS networks in elongation, and the half-filled circles to the present results in compression. Both sets of results pertain to $25^{\circ} \mathrm{C}$ and to unswollen networks having degrees of crosslinking characterized by $v_{2, \mathrm{~N}}=0.09$.

sion are very much smaller than those obtained for either unswollen ${ }^{9}$ or swollen ${ }^{10}$ PMDS networks in elongation, and are essentially independent of $v_{2, \mathrm{c}}$. These features of the stress-strain isothems in compression make the use of these results in evaluating the theories of rubberlike elasticity much less ambiguous than those previously reported $^{7,9,10}$ for this purpose.

We consider first, however, the possible correlation of the quantity $2 C_{2}$ with the nonequilibrium characteristics of the networks, as measured by the average time $t_{\mathrm{eq}}$ required for a network to reach elastic equilibrium and the extent to which the initial value of the reduced force decreases in reaching its equilibrium value. Values of these latter two quantities are given in the last two columns of Table I. Decrease in $v_{2, \mathrm{C}}$ at constant $v_{2, \mathrm{~N}}$ is seen to have no significant effect on $2 C_{2}$, but to decrease dramatically the importance of the nonequilibrium properties. Furthermore, an increase in $v_{2, \mathrm{~N}}$ at constant $v_{2, \mathrm{C}}$ increases $2 C_{2}$ but decreases the nonequilibrium effects. There is thus no correlation between $2 C_{2}$ and the length of time and the amount of stress relaxation required to reach elastic equilibrium. As has been concluded elsewhere, ${ }^{9,10,15,18}$ the $2 C_{2}$ contribution to the reduced force cannot therefore be primarily due to difficulties in reaching elastic equilibrium.

Interpretation of the results in terms of the 
Table II. Values of $\left[f^{*}\right]_{\mathrm{S}} /\left[f^{*}\right]_{\mathrm{B}}$ and $\left(2 C_{1}\right)_{\mathrm{S}} /\left(2 C_{1}\right)_{\mathbf{B}}$ for PDMS Networks in Compressiona

\begin{tabular}{|c|c|c|c|c|c|}
\hline \multirow{2}{*}{$\left(v_{2, \mathrm{C}}\right)_{\mathrm{S}}$} & \multirow{2}{*}{$v_{2, \mathrm{~N}}$} & \multicolumn{2}{|c|}{$\left[f^{*}\right]_{\mathrm{S}} /\left[f^{*}\right]_{\mathrm{B}}$} & \multirow{2}{*}{$\begin{array}{l}\left(2 C_{1}\right)_{\mathrm{S}} / \\
\left(2 C_{1}\right)_{\mathrm{B}}\end{array}$} & \multirow{2}{*}{$\begin{array}{c}{\left[f^{*}\right]_{\mathrm{S}} /\left[f^{*}\right]_{\mathrm{B}},} \\
\text { Flory Theory }\end{array}$} \\
\hline & & $\alpha=0.80$ & $\alpha=1.00$ & & \\
\hline \multirow[t]{2}{*}{0.554} & 0.08 & 1.12 & 1.16 & 1.24 & 1.05 \\
\hline & 0.11 & 1.15 & 1.19 & 1.48 & 1.06 \\
\hline \multirow[t]{2}{*}{0.428} & 0.08 & 1.20 & 1.23 & 1.33 & 1.08 \\
\hline & 0.11 & 1.15 & 1.19 & 1.40 & 1.10 \\
\hline \multirow[t]{2}{*}{0.370} & 0.08 & 1.16 & 1.15 & 1.28 & 1.10 \\
\hline & 0.11 & 1.13 & 1.21 & 1.52 & 1.13 \\
\hline \multirow[t]{2}{*}{0.269} & 0.08 & 1.19 & 1.22 & 1.30 & 1.16 \\
\hline & 0.11 & 1.21 & 1.25 & 1.63 & 1.21 \\
\hline
\end{tabular}

a Studied unswollen at $25^{\circ} \mathrm{C}$.

${ }^{b}\left[f^{*}\right]_{\mathrm{S}} /\left[f^{*}\right]_{\mathrm{B}}$ extrapolated to $\alpha^{-1}=0.0$.

volume dependence of the reduced force was carried out by plotting $\left[f^{*}\right]$ against $v_{2, \mathrm{~N}}$ for each series of networks characterized by a particular average value of $v_{2, \mathrm{c}}$, as described earlier in this section. Interpolation and limited extrapolation were then used to obtain experimental values of $\left[f^{*}\right]_{\mathrm{S}} /\left[f^{*}\right]_{\mathrm{B}}$ at values of $\alpha$ and $v_{2, \mathrm{~N}}$ judged to be most reliable on the basis of the range of these variables covered experimentally: $\alpha=0.800$ and $v_{2, \mathrm{~N}}=0.08$ and 0.11 . The illustrative results thus obtained for each of the four values of $\left(v_{2, \mathrm{c}}\right)_{\mathrm{S}}$ characterizing the networks cross-linked in solution are shown in the third column of Table II. Values of $\left[f^{*}\right]_{\mathrm{S}} /\left[f^{*}\right]_{\mathrm{B}}$ extrapolated to $\alpha=\alpha^{-1}=$ 1.000 are shown in the following column; they differ but little from those pertaining to $\alpha=$ 0.800 , as could be anticipated from the relatively small values of $2 C_{2}$ characterizing these networks in compression. Values of the ratio $\left(2 C_{1}\right)_{\mathrm{S}} /\left(2 C_{1}\right)_{\mathrm{B}}$, obtained by extrapolations of $\left[f^{*}\right]$ to $\alpha^{-1}=0.0$ on the basis of eq 3 , are shown in the fifth column of the table. Theoretical values of the ratio, calculated from the Flory theory ${ }^{8}$ for the chosen values of $v_{2, \mathrm{C}}$ and $v_{2, \mathrm{~N}}{ }^{7-10}$ are given in the final column; the corresponding values from the James and Guth theory ${ }^{14}$ would be unity throughout. In both theories, the reduced forces should be independent of $\alpha$ itself. The experimental values of this crucially important quantity at any of these values of $\alpha$ invariably exceed unity by an amount well beyond the experimental errors in such measurements, in direct contradiction to the predictions of the theory of James and Guth. ${ }^{14}$ The agreement between these experimental results and the results predicted from the Flory theory can be considered to be quite satisfactory when one takes into account the experimental difficulties in such measurements and the still poorly understood dependence of the properties of a polymer network on the presence of chain entanglements. ${ }^{7}$ The largest differences between the present experimental results and the predictions of the Flory theory ${ }^{8}$ occur in the case of the ratio defined in terms of the constant $2 C_{1}$. It is important to note in this regard that a considerably longer extrapolation is required to extract values of $2 C_{1}$ from stress-strain isotherms in compression than is the case in elongation. Also, the significance of this constant in compression measurements is particularly obscure, since it must in general differ from that characterizing the same network in elongation; the elongation and compression isotherms must intersect at $\alpha=1.0$ and, as shown here and elsewhere, ${ }^{2,3}$ isotherms in the region of compression generally have much smaller slopes (values of $2 C_{2}$ ) than do those in elongation.

Results of similar experiments ${ }^{7,9,10}$ carried out on networks in elongation are summarized and reevaluated in Table III. The previously reported experimental values of $\left[f^{*}\right]_{\mathrm{S}} /\left[f^{*}\right]_{\mathrm{B}}$ at elongations of $\alpha=1.3$ and 1.7 are given in columns four and five; in addition, these results are reevaluated in that values of $\left(2 C_{1}\right)_{\mathrm{S}} /\left(2 C_{1}\right)_{\mathrm{B}}$ are now also presented, in column six. The last column lists the values predicted by the Flory theory. ${ }^{7-10}$ In the case of the cis-1,4-polybutadiene networks, the experimental values of $\left[f^{*}\right]_{\mathrm{s}} /$ $\left[f^{*}\right]_{\mathrm{B}}$ are well below unity. This unexpected result may be at least partially due to inhomogeneties in cross-linking resulting from the preparation of these networks at relatively low polymer concentrations $\left(v_{2, \mathrm{C}} \leqslant 0.10\right)$, as was suggested previously. ${ }^{7}$ The observation, however, that values of $\left(2 C_{1}\right)_{\mathrm{S}} /\left(2 C_{1}\right)_{\mathrm{B}}$ obtained from this study do exceed unity and are in reasonable agreement with the Flory predictions suggests that a major source of the disagreement in the case of the values of $\left[f^{*}\right]_{\mathrm{S}} /\left[f^{*}\right]_{\mathrm{B}}$ at $\alpha=1.3$ and 1.7 may have been due to the fact that the values of $2 C_{2}$ for these cis-1,4-polybutadiene networks in elongation are unusually large $(0.02-$ $0.20 \mathrm{~N} \mathrm{~mm}^{-2}$ ) and the networks prepared in solution exhibited much smaller values of $2 C_{2}$ than did the networks prepared in the undiluted state. 
Table III. Summary and Reevaluation of Previous Results Bearing on the Volume Dependence of the Elastic Free Energy

\begin{tabular}{|c|c|c|c|c|c|c|}
\hline \multirow{2}{*}{ Polymer Networks ${ }^{a}$} & \multirow{2}{*}{$\left(v_{2, \mathrm{C}}\right)_{\mathrm{s}}$} & \multirow{2}{*}{$v_{2, \mathrm{~N}}$} & \multicolumn{2}{|c|}{$\left[f^{*}\right]_{\mathrm{S}} /\left[f^{*}\right]_{\mathrm{B}}$} & \multirow{2}{*}{$\left(2 C_{1}\right)_{\mathrm{S}} /\left(2 C_{1}\right)_{\mathrm{B}}^{\mathrm{b}}$} & \multirow{2}{*}{$\begin{array}{l}{\left[f^{*}\right]_{\mathrm{s}} /\left[f^{*}\right]_{\mathrm{B}}} \\
\text { Flory Theory }\end{array}$} \\
\hline & & & $\alpha=1.3$ & $\alpha=1.7$ & & \\
\hline \multirow{2}{*}{$\begin{array}{l}\text { Cis-1,4-Polybutadiene, } \\
\text { unswollen }\end{array}$} & 0.09 & 0.09 & 0.765 & 0.834 & 1.55 & 1.80 \\
\hline & 0.06 & 0.06 & 0.516 & 0.596 & 2.53 & 1.85 \\
\hline \multirow{12}{*}{$\begin{array}{l}\text { Poly(dimethylsiloxane), } \\
\text { unswollen }{ }^{9}\end{array}$} & 0.69 & 0.09 & 1.04 & 1.03 & 1.05 & 1.02 \\
\hline & & 0.12 & 1.05 & 1.03 & 1.15 & 1.03 \\
\hline & 0.58 & 0.09 & 1.07 & 1.06 & 1.01 & 1.04 \\
\hline & & 0.12 & 1.09 & 1.08 & 1.27 & 1.05 \\
\hline & 0.52 & 0.09 & 1.08 & 1.07 & 1.10 & 1.06 \\
\hline & & 0.12 & 1.12 & 1.13 & 1.41 & 1.07 \\
\hline & 0.45 & 0.09 & 1.09 & 1.08 & 1.03 & 1.08 \\
\hline & & 0.12 & 1.10 & 1.12 & 1.42 & 1.09 \\
\hline & 0.38 & 0.09 & 1.04 & 1.07 & 1.31 & 1.10 \\
\hline & & 0.12 & 1.05 & 1.09 & 1.67 & 1.13 \\
\hline & 0.28 & 0.09 & 1.03 & 1.07 & 1.41 & 1.17 \\
\hline & & 0.12 & 1.01 & 1.08 & 1.85 & 1.21 \\
\hline \multirow{12}{*}{$\begin{array}{l}\text { Poly(dimethylsiloxane), } \\
\text { swollen }^{10}\end{array}$} & 0.69 & 0.09 & 1.07 & 1.06 & 1.06 & 1.02 \\
\hline & & 0.12 & 1.04 & 1.06 & 1.13 & 1.03 \\
\hline & 0.58 & 0.09 & 1.11 & 1.11 & 1.20 & 1.04 \\
\hline & & 0.12 & 1.09 & 1.10 & 1.18 & 1.05 \\
\hline & 0.52 & 0.09 & 1.14 & 1.14 & 1.19 & 1.06 \\
\hline & & 0.12 & 1.17 & 1.19 & 1.38 & 1.07 \\
\hline & 0.45 & 0.09 & 1.16 & 1.16 & 1.25 & 1.08 \\
\hline & & 0.12 & 1.16 & 1.20 & 1.34 & 1.09 \\
\hline & 0.38 & 0.09 & 1.19 & 1.21 & 1.32 & 1.10 \\
\hline & & 0.12 & 1.13 & 1.19 & 1.59 & 1.13 \\
\hline & 0.28 & 0.09 & 1.18 & 1.21 & 1.59 & 1.17 \\
\hline & & 0.12 & 1.08 & 1.15 & 1.37 & 1.21 \\
\hline
\end{tabular}

a Studied in elongation, at $25^{\circ} \mathrm{C}$.

${ }^{\text {b }}\left[f^{*}\right]_{\mathrm{S}} /\left[f^{*}\right]_{\mathrm{B}}$ extrapolated to $\alpha^{-1}=0.0$.

In the case of both the unswollen and swollen PDMS networks in elongation, all of the results support the Flory theory as being the more nearly correct. $^{19}$ The experimental values of the ratio are not only in at least approximate agreement with the values calculated from the Flory theory, but also generally increase with decreasing $\left(v_{2}, \mathrm{c}\right)_{\mathrm{S}}$ and increasing $v_{2, \mathrm{~N}}$, as predicted. ${ }^{7-10}$

The results of the present study, in conjunction with those previously reported, ${ }^{7,9,10,19}$ thus definitively settle what had been one of the most controversial questions in the area of rubberlike elasticity.

Acknowledgements. Acknowledgement is made to the donors of the Petroleum Research Fund, administered by the American Chemical Society, for partial support of this work. C. U.Y. also wishes to acknowledge, with gratitude, the predoctoral fellowship generously provided by the Macromolecular Research Center of the University of Michigan.

\section{REFERENCES}

1. L. R. G. Treloar, "The Physics of Rubber Elasticity," 2nd ed, Clarendon Press, Oxford, 1958.

2. Some typical studies of polymer networks in compression are cited in ref 3.

3. R.Y.S. Chen, C. U. Yu, and J.E. Mark, Macromolecules, 6, 746 (1973).

4. A number of investigations of the properties of polymer networks prepared in solution are cited in ref 5 .

5. J. E. Mark, Rubber Chem. Technol., 46, 593 (1973).

6. Reference 7 provides a list of references to 
papers in which the molecular theories of rubberlike elasticity are developed, and differences between them discussed in detail.

7. J. E. Mark, J. Amer. Chem. Soc., 92, 7252 (1970).

8. See, for example, P. J. Flory, Trans. Faraday Soc., 57, 829 (1961).

9. R. M. Johnson and J. E. Mark, Macromolecules, 5, 41 (1972).

10. C. U. Yu and J. E. Mark, ibid., 6, 751 (1973).

11. M. Mooney, J. Appl. Phys., 11, 582 (1940); R. S. Rivlin, Phil. Trans. Roy. Soc. London, Ser. A, 240, 459, 491, 509 (1948).

12. W. R. Krigbaum and R.-J. Roe, Rubber Chem. Technol., 38, 1039 (1965).

13. L. R. G. Treloar, Rep. Progr. Phys., 36, 755 (1973).

14. See, for example, H. M. James and E. Guth, J. Chem. Phys., 21, 1039 (1953).
15. K. Dušek and W. Prins, Advan. Polym. Sci., 6, 1 (1969).

16. D. Froelich, D. Crawford, T. Rozek, and W. Prins, Macromolecules, 5, 100 (1972).

17. C. U. Yu, Ph. D. Thesis in Chemistry, The University of Michigan, Ann Arbor, Michigan, 1974.

18. J. E. Mark and P. J. Flory, J. Appl. Phys., 37, 4635 (1966).

19. Prins and coworkers also reach the conclusion that the Flory theory is the more nearly correct, on the basis of measurements carried out on elastomeric polystyrene networks; see ref 16 . Their analysis, however, requires a number of assumptions regarding details of the network structure that are difficult, if not impossible, to assess. 